\title{
Energy Tunneling Behavior in Geometrically Separated Wave Guides
}

\author{
Muhammad Omar ${ }^{1}$, Rashad Ramzan ${ }^{1}$, Omar Farooq Saddiqui ${ }^{2}$ \\ ${ }^{1}$ Department of Electrical Engineering, UAE University, Alain-15551, UAE \\ ${ }^{2}$ College of Engineering, Taibah University, University Road, Madinah, Saudi Arabia \\ *corresponding author, E-mail: muhammadomar@uaeu .ac . ae
}

\begin{abstract}
In this paper, characteristics of energy tunneling channel between the waveguides geometrically separated by a coaxial cable are studied. The novel aspect of design is use of coaxial channel to connect the waveguides while maintaining the energy tunneling phenomena. As anticipated the tunneling frequency depends upon the length of wire inside the waveguide and the length of the coaxial cable. The tunneling frequency also depends upon the dielectric constant of the material inside the waveguide and coaxial cable. At tunneling frequency the field strength (E and $\mathrm{H}$ ) in the channel is extremely high, making the channel extremely sensitive to small change in permittivity of dielectric occupying the channel. The advantage of the proposed design is, its ability to tune to desired tunneling frequency just by changing the length of the coaxial cable without the need to redesign the waveguide height to accommodate the long tunneling wires. This structure can be used as dielectric sensor both for solid or liquid dielectrics just by placing the sample in coaxial cable cavity, contrary to previously report work where the sample has to be placed inside the waveguide.
\end{abstract}

\section{Introduction}

The electromagnetic energy tunneling which takes place in narrow channels and bends loaded with epsilon-near-zero (ENZ) materials or resonant wires is accompanied by very intensive electric fields [1]-[3]. So far, a new class of microwave components like multiplexers, filters, and dielectric sensors has been demonstrated experimentally exploiting the energy tunneling phenomena [4]-[6]. Energy tunnels through narrow sub-wave length channel filled with ENZ materials inherits high dielectric loses at low frequencies [7], and to avoid these loses they have to operate at cutoff frequency which solely depends upon the geometry of the waveguide. To tune these systems at desired frequency one have to redesign the whole waveguide hence it is not possible to operate the same waveguide design filled with ENZ materials at different or multiple tunneling frequency. On the other hand, in the wire loaded energy tunneling designs, energy tunnels through the metallic wire placed inside the waveguide. The tunneling frequency mainly depends upon the length of the wire. An intense electric field is created at the surface of these

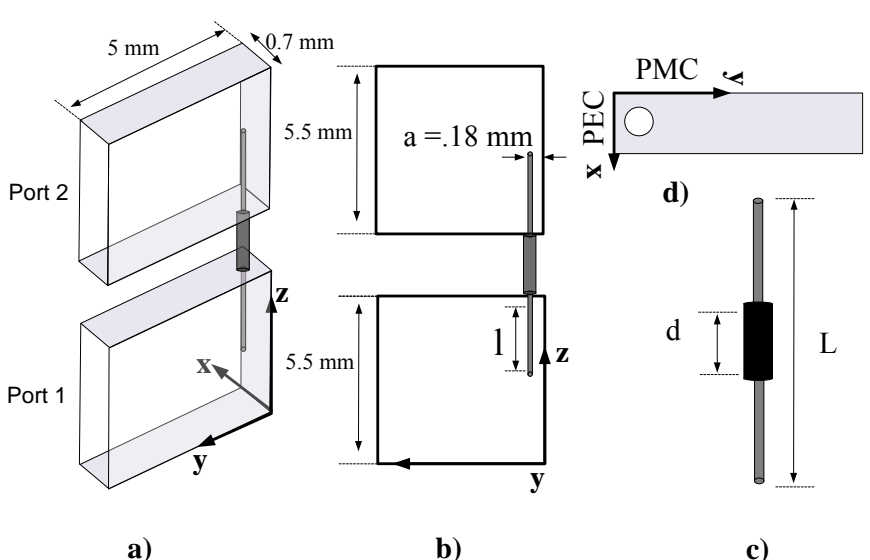

Fig.1. (a) Waveguides couples through cylindrical channel, shaded area represents PEC boundaries and unshaded area represents PMC boundaries (b) side view; (c ) metallic wire and channel between waveguides; (d) top View, location of aperture.

metallic wires, which is extremely sensitive to the dielectric material around it. This effect is exploited to design very sensitive dielectric sensor [8]-[9]. In order to design the robust and accurate dielectric sensors of different kinds, the detail and in depth study of tunneling channel and its characteristics is mandatory. The second equally important factor is the shape and physical size of the energy tunneling channel when used as a sensor. This is important in order to convenient placement and removal of sample in the channel.

In this work, we have proposed the use of coaxial channel to connect the two waveguides with each other while maintaining the energy tunneling. Now, in this case, the coaxial cable segment has a potential to be used a sample holder both for solid and liquid dielectric samples. Moreover, the tunneling frequency can be programed at desired value just by changing the length of the coaxial cable, without changing the design of the waveguide. Thus alleviating the geometrical constraint that the tunneling frequency is determined by the length of wire and that wire cannot be longer than the height of the waveguide.

The geometry of the proposed design is shown in Fig. 1 (a)(d). Coaxial cable is used as a narrow cylindrical channel where the core of coaxial cable is used as metallic tunneling wire. Both waveguides are geometrically separated through coaxial channel as shown in Fig 1.a. The side view of model 
is shown in Fig 1. (b). In this present situation, the tunneling frequency of metallic wire embedded inside the waveguide is $\mathrm{L}=21+\mathrm{d}$, where 1 is the length of wire protruding inside waveguide and $d$ is the length of coaxial cable, as show in Fig. 1.c. The coaxial cable acts as narrow cylindrical channel or transmission line. The left and right walls of waveguides are taken as perfect magnet conductor (PMC) whereas the back side wall is taken as perfect electrical conductor (PEC), as shown in Fig. 1.d. The dimensions of channel are selected such that, it permits the electromagnetic energy to tunnel through it at tunneling frequency without any loss. If the dimensions of the waveguides are such that they only supports dominate transvers electric $\left(\mathrm{TE}_{10}\right)$ mode, then it is very challenging to connect them with source especially when both waveguides are connected at $180^{\circ}$ bend. By coupling waveguides through coaxial cable, it is very easy to connect the waveguides to the source. As coaxial cable inherit flexibility in structure and can be twist and bend.

Moreover, in this proposed design, we can rotate, bend, and twist the channel without redesigning the whole system of waveguide as shown in Fig. 2. Hence, different arrangements of the channel connections reported in [9] can be achieved without redesigning the whole system. This will also allow the flexibility in the shape and size of the dielectric sample when this structure is used as a sensor.

The paper is divided as follows. In section 2, the proposed structure is simulated using ANSOFT'S ${ }^{\circledR}$ HFSS, and simulation results are presented along with comparison with recent LNAs and the paper is finally concluded in Section 3.

\section{SIMULATION RESULTS}

The design mentioned in Fig.1, is simulated and results are reported. The channel dimension is selected such that there is no energy tunneling in the absence of wire. It can be clearly seen from the plot in Fig. 3 that no energy tunnels from waveguide- 1 to waveguide- 2 when no any wire is placed inside waveguides.

$$
\varepsilon_{r}=1-\frac{\omega^{2}}{\omega_{p}^{2}}
$$

In the presences of metallic wire, when operational frequency becomes equal to the plasma frequency of wire then according to equation (1) effective permittivity $\left(\mathcal{E}_{\mathrm{r}}\right)$ becomes zero which force the phase velocity to become infinite and hence energy couples from one waveguide to other with perfectly matched condition.

In Fig.3, two peaks are noticed. The lower frequency peak is due to the wire embedded inside the waveguide and its tunneling frequency depends upon the wire length and channel length i.e., $\mathrm{L}=2 \mathrm{l}+\mathrm{d}$. Whereas the second frequency is because of Fabry-Perot resonance and depends upon the dimensions of the channel connecting them, polyethylene material ( $\mathcal{E}_{r}=2.25$ ) is used as dielectric between core and cladding of coaxial cable.

As there is no any energy tunneling, in the absence of wire and Fabry-Perot resonance only depends on the channel

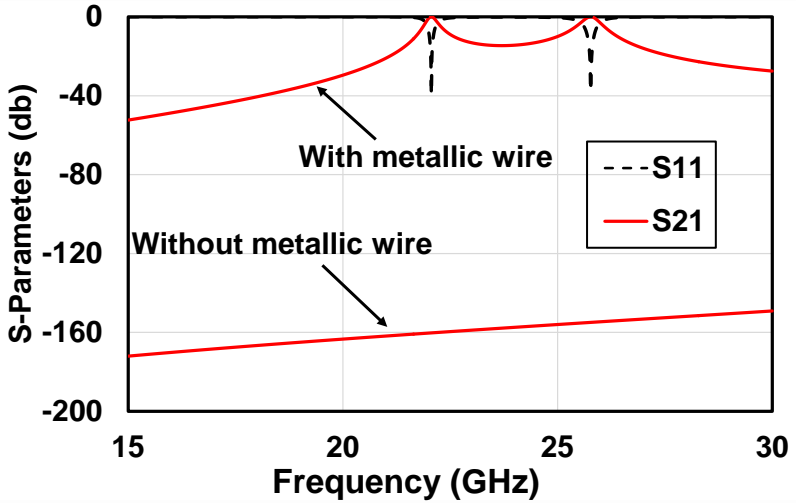

Fig.3. S-parameters with and without metallic wire

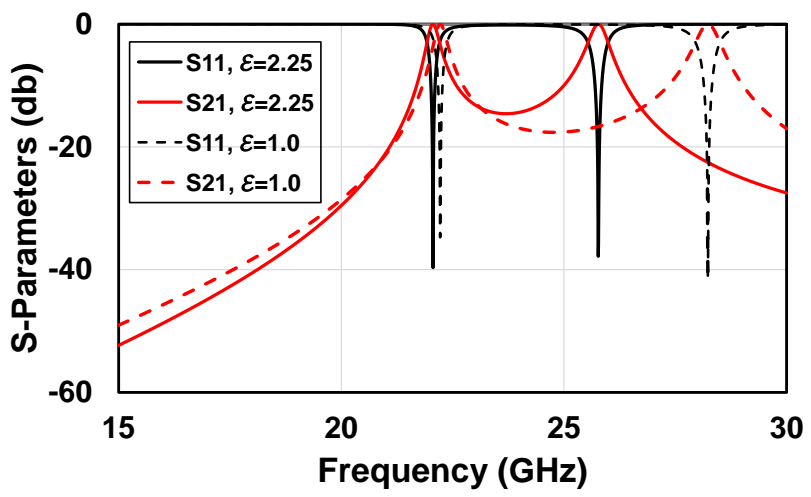

Fig.4. Effect of permittivity on S-parameter

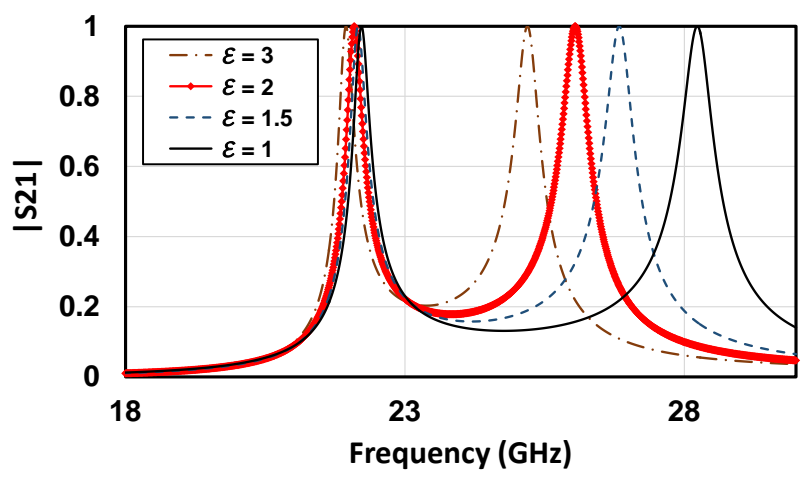

Fig.5. Effect of permittivity change from 1-3, on magnitude of transmission coefficient

dimensions. If this approach is use then, we can tune the higher frequency peak too and even channel can be used for sensing. As it is mentioned that higher frequency peak only depends upon the channel dimensions, and by connecting the waveguides as mentioned in Fig 1. The coaxial cable can be used to place the dielectric sample. To study the characteristic of channel, the waveguide is simulated with different permittivity and channel lengths. The $S_{11}$ and $S_{22}$ parameters are given in Fig. 4 when channel is loaded with different dielectric materials. Curve represented with dashedline is taken when coaxial cable is loaded with dielectric 
material having permittivity 1 and solid line is used to represent S-parameters for dielectric material with permittivity 2.25 . A large frequency shift of $3 \mathrm{GHz}$ is noticed when channel is loaded with different dielectric samples. It can be seen from the plots that only higher frequency peak shifts and the position of the lower frequency peak on frequency scale is almost the same. A very small shift in the lower frequency peak can be attributed to the fact that placing the tunneling wire inside the channel changes the dimensions of the channel. The slight change in the effective volume of the waveguide channel causes the lower frequency peak to move slightly.

Similarly, magnitude plot when channel is loaded with different dielectric materials is plotted in Fig.5. Different dielectric materials are used with permittivity range from 1 to 3. It can be seen from figure that only higher frequency peak shifts and lower frequency peak is almost constant. If we increase the permittivity then after a certain range behavior of both peaks starts to change and for permittivity above 5, the shift in higher frequency peak slowly drop down and lower frequency peak starts to move with change in the permittivity of material filling the channel.

The comparison of change in the tunneling frequency with change in permittivity of sample is given in Fig. 7. It can be seen from the figure that for permittivity below 5 , higher frequency peak shows more sensitivity then first and for permittivity ranges above 5 lower frequency peak shows more sensitivity then the higher frequency peak. As it discussed before that lower frequency peak depends on channel length too. If we increase the channel length $d$ to a level that it becomes comparable with length 21 , then first resonance peak will also shift with higher frequency resonance peak. This property is very important and makes the sensor practically more flexible as operating frequency of the sensor can be adjust just by using large length of coaxial cable. The shift in tunneling frequencies with change in effective channel length is shown in Fig. 8. And due to this property we can tune the sensor at any desired lower frequency. If the first resonance peak is to be used for dielectric sensing, then length of the channel must be selected such that any change in channel length should be realized using the $\mathrm{L}$ and length $\mathrm{d}$. As length $\mathrm{d}$ is also very sensitive to change in in its close proximity, hence change in permittivity also affect the effective length $\mathrm{d}$. If $\mathrm{d}$ is selected to be very small; approximately equal to zero, then the tunneling frequency only depends upon the length $\mathrm{L}=21$. In that case, we are only left with lower frequency. The effect of change in tunneling frequency with change in length 1 , is plotted in Fig. 9.

It is recommended that for better sensitivity, the dielectric sample is placed inside the channel rather than inside the waveguide. It is very easy to place dielectric sample inside the channel especially when, waveguide is filled with dielectric material other than air. It is also possible to inject liquid dielectric samples like, Toluene $(\varepsilon=2.0-2.4)$, Bromine ( $\mathcal{E}=3.1)$, and Chlorine $(\mathcal{E}=2)$ and cresol $(\mathcal{E}=10.6)$ i.e., into the coaxial cable channel especially designed to hold the liquid samples. In this way we do not need to open the wave guide to place the sample, we can put the sample inside coaxial

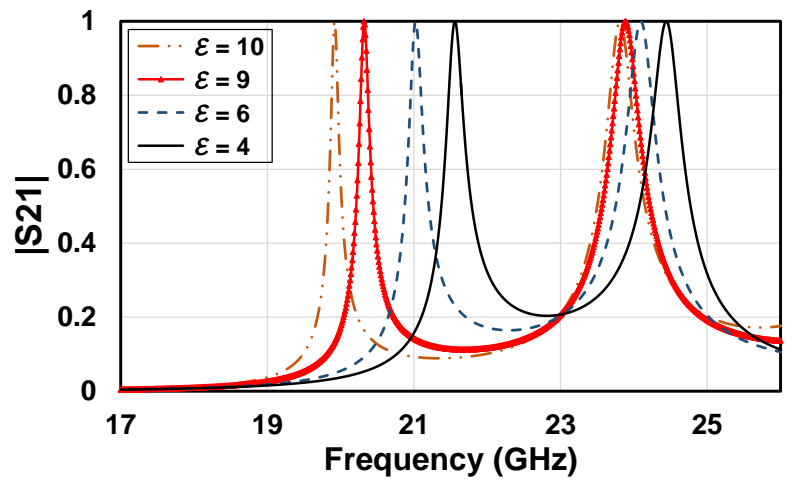

Fig.6. Effect of permittivity change from 4-10, on magnitude of transmission coefficient

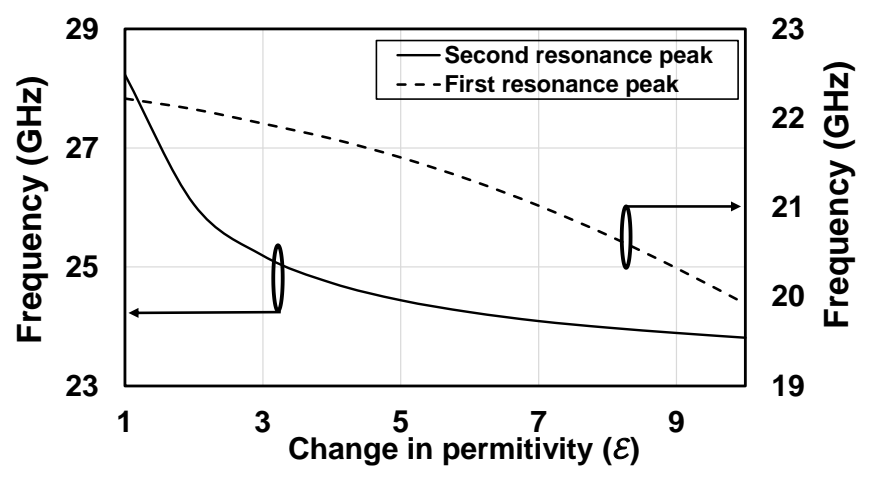

Fig.7. Effect of permittivity on sensitivity of resonance peaks

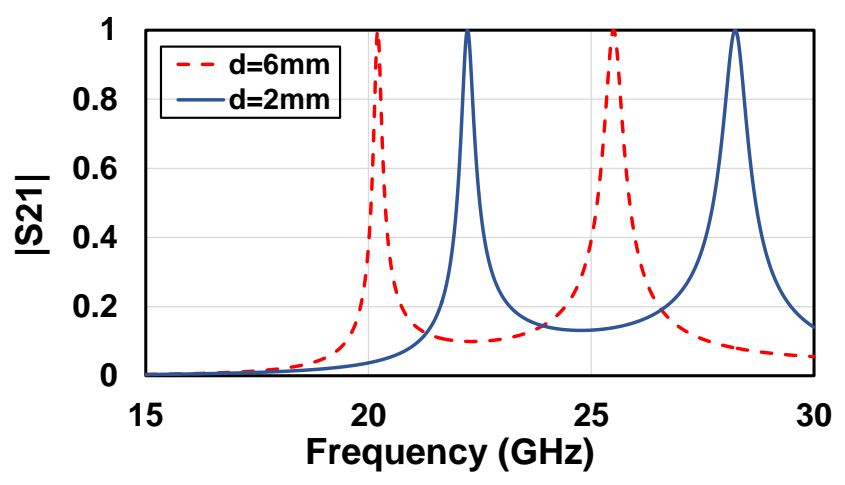

Fig.8. Effect of change in length coaxial cable on magnitude of transmission coefficient

cable, replacing the coaxial dielectric with liquid dielectric sample under test and can use the solid, liquid and gas samples.

The Energy through primary waveguide can be further divide into N-number of waveguides as shown in Fig. 10. In that case power delivered to each waveguide will be $\mathrm{P}_{\text {in }} / \mathrm{N}$, where Pin launched inside waveguide 1 . The power divider is used to distribute equal power between waveguides, as shown in Fig.10 (b). Equal power is delivered to both waveguides as plotted in Fig. 11. The maximum magnitude of transmission coefficient for each waveguide is 0.5 or $\mathrm{P}_{\text {in }} / 2$ and can be 


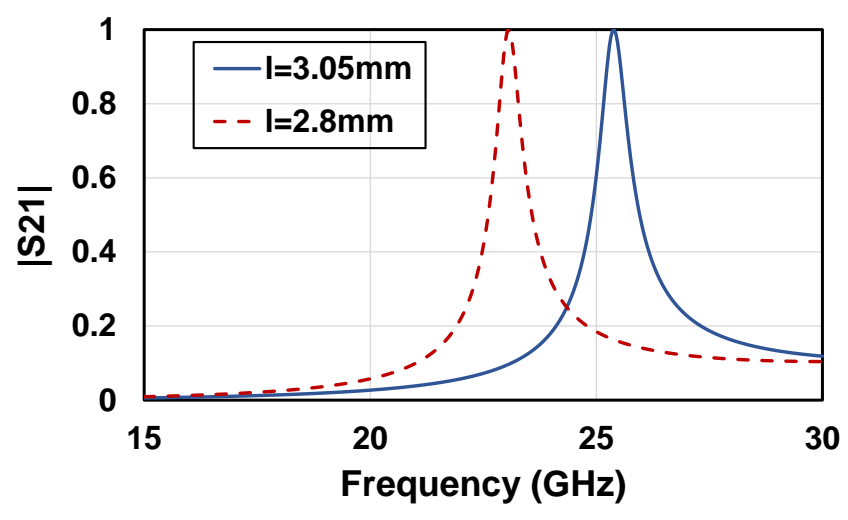

Fig.9. Effect of change in length of metallic wire on magnitude of transmission coefficient

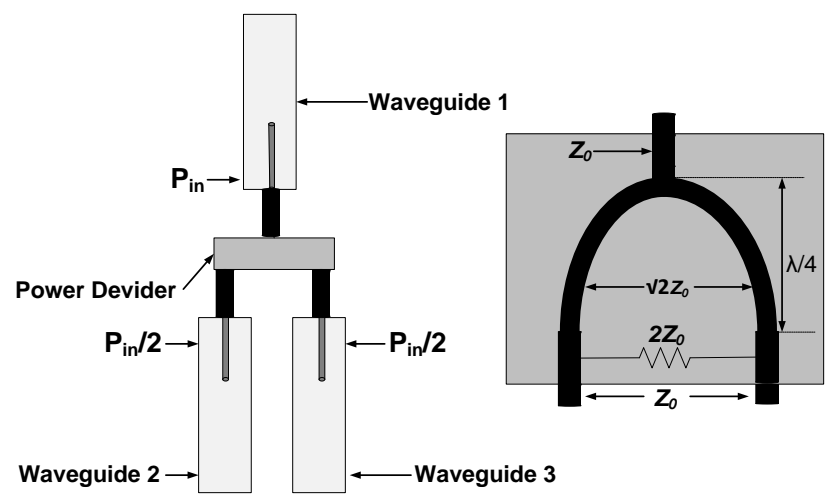

a)

b)

Fig10. a) Power further divides to different waveguides; b) construction of power divider
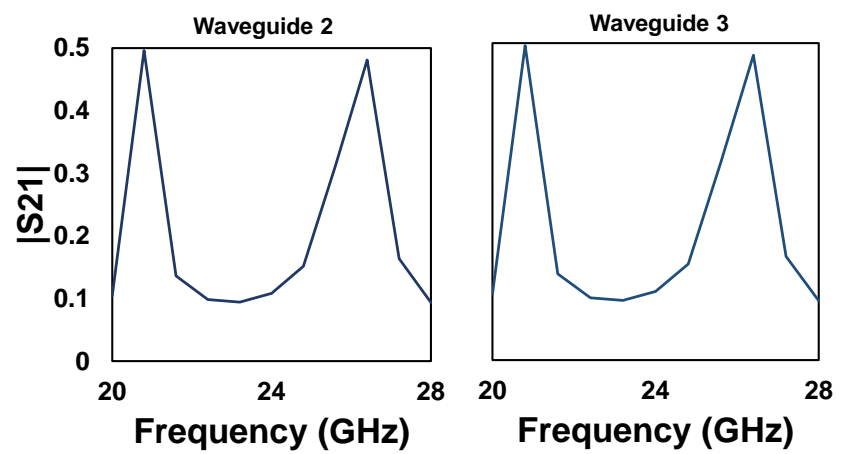

Fig11. Magnitude of transmission coefficient at each waveguide

extend to N-number of waveguides. This means that the either multiple sensors can be operate at same time at same frequency using the same waveguide. It is also possible that multiple sensor can work at different frequencies without changing the dimensions of waveguides.

\section{Conclusion and Future Directions}

The characteristics of energy tunneling channel in wire loaded waveguides was studied and new design was proposed in which coaxial cable was used for coupling of waveguides. The effect of permittivity of the dielectric sample placed inside the coaxial cable on the characteristic channel is studied and reported. It is shown that such configurations has a potential to be used an extremely sensitive sensor for solid, liquid and gas dielectrics. The use of power divide in coaxial cable channel open the new possibilities for sensor to work at different frequencies using the same waveguide structure, which is our future direction of work.

\section{Acknowledgment}

This is work is supported by UAE University Grant: $31 \mathrm{~N} 206$.

\section{References}

[1] A. Alu and N. Engheta, "Light squeezing through arbitrarily shaped plasmonic channels and sharp bends," Phys. Rev. B, vol. 78, p. 035440, Jul. 2008.

[2] A. Alu, M. Silveirinha, and N. Engheta, "Transmission-line analysis of epsilon-near-zero (ENZ)-filled narrow channels," Phys. Rev. E, vol. 78, p. 016604, Jul. 23, 2008.

[3] M. G. Silveirinha and N. Engheta, "Theory of supercoupling, squeezing wave energy, and field confinement in narrow channels and tight bends using nearzero metamaterials," Phys. Rev. B., vol. 76, no. 24, p. 245109, Dec. 2007.

[4] Omar Siddiqui, Mani Kashanianfard and Omar Ramahi,"Dielectric Sensors Based on Electromagnetic Energy Tunneling" Sensors, 15, 7844-7856, 2015.

[5] A. Nauroze1, O. Sidiqui, R. Ramzan, and O. Ramahi, "Dielectric Sensing based on Energy Tunneling in Wireloaded Microstrip Cavities" META'13, 18-22, Sharjah , UAE, March 2013.

[6] O. F. Siddiqui and O. M. Ramahi, "Frequency-selective energy tunneling in wire-loaded narrow waveguide channels," PIER, Letters, Vol. 15, 153-161, 2010.

[7] Alu A.-Young-M.E. Silveirinha M.-Engheta N. Edwards, B. "Experimental veri_cation of epsilon-near-zero metamaterial coupling and energy squeezing using a microwave waveguide," Physical Review Letters, 100(3), 2008.

[8] R. Ramzan, O. Siddiqui, A. Nauroze, and O. Ramahi," Microstrip Probe Based on Electromagnetic Energy Tunneling for Extremely Small and Arbitrarily Shaped Dielectric Samples" IEEE antennas and wireless propagation letters, vol. 14, 2015.

[9] M. Kashanianfard, "Electromagnetic Wave Transmission through Sub-wavelength Channels and Bends Using Metallic Wires, M.S. thesis, Dept. Elect. And Comp. Eng., Univ. of Waterloo, Waterloo, Ontario, Canada, 2009. 